$52 \%$ and limb swelling in 51.4\%. Pallor indicative of anaemia is seen in all $(100 \%)$ of patients, tachycardia seen in $91 \%$ and hepatomegaly detected in $63.8 \%$. Growth failure observed in $51.45 \%$. All study population had low haemoglobin, ranging from 2.9 - $9.5 \mathrm{gm} / \mathrm{dl}$ with a mean $7.01 \mathrm{gm} / \mathrm{dl}$ ( $\pm 1.1 \mathrm{SD})$, Reticulocytosis observed in $18.8 \%$ and Leucocytosis in $85.5 \%$. Ninety percent recovered from their acute episodes, (5\%) were referred to other hospital, only three patients $(2.2 \%)$ leaved against medical advice, two patients (1.4\%) discharged home with complications, there were two deaths (1.4\%).

Conclusions The study exemplifies different patterns of presentations in patients with Sickle cell disease with variable percentage. It is suggested to manage considering that patterns and applying the multi-disciplinary approach.

\section{MICROBIAL PATTERNS AND ANTIBIOTIC SUSCEPTIBILITY IN BLOOD CULTURE ISOLATES OF SEPTICEMIA SUSPECTED CHILDREN IN THE PEDIATRICS WARD OF A TERTIARY CARE HOSPITAL}

Muhammad Sarfraz Khan, Arslan Kareem, Areeb Khalid, Saima Rauf, Muhammad Shaheryar Bashir, Kiran Fatima. Pakistan

\subsection{6/bmjpo-2021-RCPCH.40}

Background Septicemia is considered as the second most common cause of death in non-coronary intensive care units (ICU) and tenth overall cause of death in countries with high per capita income. Early detection and determination of antimicrobial susceptibility patterns have been shown to reduce the morbidity and mortality associated with bloodstream infections.

Objectives This study aims to determine microbial patterns and antibiotic susceptibility to alert clinicians to the emerging pathogens that may pose a threat to the community, especially in children.

Methods This retrospective cross-sectional study was conducted in the Department of Pathology, Holy Family Hospital, Rawalpindi, Pakistan, from July 2019 to December 2019. The culture was performed on blood and MacConkey agar. Microbes were identified under a microscope by observing their morphological characteristics after gram staining and applying biochemical tests. Antibiotic sensitivity test was carried out using standard aseptic methods. Bacterial isolates and their susceptibility patterns were represented using frequencies and percentage charts.

Results Out of 423 blood cultures, growth was recorded in 92 $(21.75 \%)$ of the cultures with female to male ratio $2.1: 1$. The gram-positive bacteria accounted for $43.48 \%(n=40)$ whereas gram-negative bacteria covered the majority $54.36 \% \quad(n=50)$. Among isolates, Staphylococcus aureus (42.39\%) was the most common, followed by Acinetobacter spp. (17.39\%) and Pseudomonas aeruginosa (14.13\%). Acinetobacter spp. showed 0\% susceptibility to amikacin and cefotaxime. All the isolates were $100 \%$ resistant to amoxicillin-clavulanic acid. S. aureus showed lower sensitivity for ceftazidime (0\%), clindamycin (66.67\%), ciprofloxacin $(0 \%)$, clarithromycin $(11.76 \%)$ and ceftriaxone $(0 \%)$. Tigecycline showed $100 \%$ sensitivity for all isolates tested.

Conclusions Gram-negative bacteria form the majority of isolates in our set up with Acinetobacter most common species among them. The resistance against cephalosporins, penicillin, and fluoroquinolones shown by Acinetobacter, Pseudomonas,
Salmonella, and Klebsiella is of grave concern. Among Grampositive bacteria, S. aureus has established resistance against multiple drugs. Limited and objective use of antibiotic therapy is a much-needed strategy under new guidelines.

\section{DENGUE FEVER AS A RARE CAUSE OF IMMUNE THROMBOCYTOPENIC PURPURA - A PAEDIATRIC CASE REPORT}

Nurul Ain Mohamed Ashrudin, Caroline Eng Siew Yin. Malaysia

\subsection{6/bmjpo-2021-RCPCH.4}

Background Immune thrombocytopenic purpura (ITP) typically presents with mucocutaneous bleeding and low platelet count. ITP is an autoimmune disorder characterised by autoantibody mediated immunologic destruction of normal platelets. It can be either primary or secondary due to other medical disorders. Most children have a history of preceding viral infection and tends to be self-limiting in nature. However, a minority have persistent thrombocytopenia that leads to chronic ITP.

Thrombocytopenia is a common laboratory finding in dengue fever. However, platelet count usually normalises during the recovery phase of dengue infection. Persistent thrombocytopenia requires further investigations and surveillance to ensure recovery. Here, we report a case of ITP following dengue infection responsive to corticosteroid treatment.

Objectives We aim to highlight the importance of surveillance of platelet count in Dengue infection to ensure normalcy. We also hope to create awareness of possibility of ITP when thrombocytopenia persists.

Methods A previously healthy 4 years old boy was admitted to our institution for Dengue fever, which was confirmed by a positive Dengue NS-1 Antigen testing and Dengue Immunoglobulin $\mathrm{M}$ (IgM). He was treated with intravenous fluid and serial monitoring of full blood count. His platelet count remained low at 1 to $8 \times 10^{9} / \mathrm{L}$ and failed to normalise by second week of infection. There was no history of bleeding tendencies, neither was there history of recurrent infection, joint pain, appetite and weight loss, consumption of traditional medications as well as significant family history of bleeding disorders or malignancy. The child was pink and anicteric. Lymph nodes were not enlarged and hepatosplenomegaly not present. Peripheral blood films confirmed thrombocytopenia. No blasts cells seen. Connective tissue disease screening was unremarkable. He was given a dose of intravenous immunoglobulin (IVIG) therapy. He remained asymptomatic and was then followed up as outpatient.

Results $\mathrm{He}$ presented two weeks after with petechiae and bruises after a minor fall. Upon arrival, his vital signs were stable. He had petechiae rashes and bruise over left forearm. Persistence of thrombocytopenia prompted for bone marrow aspiration and trephine biopsy (BMAT). The procedure yielded normocellular marrow with increased megakaryocytes, peripheral isolated thrombocytopenia compatible with peripheral platelet destruction. He was then started on corticosteroid. $\mathrm{He}$ showed initial good response to therapy. At the time of writing, his counts were monitored on outpatient basis.

Conclusions This case report highlights the importance of trending platelet count and ensure that they normalised after recovery phase of dengue fever. Persistent thrombocytopenia warrants further investigations to rule out secondary cause. 